\title{
Hot-carrier cooling in lead-bromide perovskite materials
}

\section{Thomas R. Hopper, Andrei A. Gorodetsky, Franziska Krieg, Maryna I. Bodnarchuk, Xiaokung Huang, et al.}

Thomas R. Hopper, Andrei A. Gorodetsky, Franziska Krieg, Maryna I. Bodnarchuk, Xiaokung Huang, Robert Lovrincic, Maksym V. Kovalenko, Artem A. Bakulin, "Hot-carrier cooling in lead-bromide perovskite materials," Proc. SPIE 11084, Physical Chemistry of Semiconductor Materials and Interfaces XVIII, 110840F (9 September 2019); doi: 10.1117/12.2528131 States 


\title{
Hot-carrier cooling in lead-bromide perovskite materials
}

\author{
Thomas R. Hopper ${ }^{\mathrm{a}}$, Andrei A. Gorodetsky ${ }^{\mathrm{a}}$, Franziska Krieg ${ }^{\mathrm{b}}$, Maryna I. Bodnarchuk ${ }^{\mathrm{b}}$, \\ Xiaokung Huang ${ }^{\mathrm{c}}$, Robert Lovrincic ${ }^{\mathrm{c}}$, Maksym V. Kovalenko ${ }^{\mathrm{b}}$, and Artem A. Bakulin ${ }^{\mathrm{a}}$ \\ ${ }^{a}$ Ultrafast Optoelectronics Group, Department of Chemistry, Imperial College London, London \\ W12 0BZ, United Kingdom \\ ${ }^{b}$ Laboratory of Inorganic Chemistry, Department of Chemistry and Applied Biosciences, ETH \\ Zurich, Vladimir-Prelog-Weg 1-5/10, 8093 Zurich, Switzerland \\ 'Institute for High-Frequency Technology, Technische Universität Braunschweig, \\ Schleinitzstrasse 22, 38106 Braunschweig, Germany
}

\begin{abstract}
Lead-halide perovskites are currently the highest-performing solution-processable semiconductors for solar energy conversion, with record efficiencies rapidly approaching that of the Shockley-Queisser limit for single-junction solar cells. Further progress in the development of lead-halide perovskite solar cells must overcome this limit, which largely stems from the ultrafast relaxation of high-energy hot carriers above the bandedge. In this contribution, we use a highly-specialized pump-push-probe technique to unravel the key parameters which control hot carrier cooling in bulk and nanocrystal (NC) lead bromide perovskites with different material composition, NC diameter and surface treatment. All samples exhibit slower cooling for higher hot carrier densities, which we assign to a phonon bottleneck mechanism. By comparing this density-dependent cooling behavior in the different samples, we find that the weak quantum confinement of electronic states and the surface defects in the NCs play no observable role in the hot carrier relaxation. Meanwhile, in accordance with our previous observations for bulk perovskites, we show that the cation plays a critical role towards carrier cooling in the perovskite NCs, as evidenced by the faster overall cooling in the hybrid $\mathrm{FAPbBr}_{3} \mathrm{NCs}$ with respect to the all-inorganic $\mathrm{CsPbBr}_{3}$ NCs. These observations highlight the crucial role of the cations toward the phononic properties of lead-halide perovskites, and further point towards the defect tolerance of these emerging solution-processed semiconductors.
\end{abstract}

Keywords: carrier cooling, hot carrier, lead-halide perovskite, nanocrystals, ultrafast spectroscopy, electronphonon coupling, charge dynamics, photophysics

\section{INTRODUCTION}

Among all the solution-processable semiconductors for solar energy conversion, lead-halide perovskites continue to make the largest strides in performance. ${ }^{1}$ The record power conversion efficiency for a single-junction perovskite solar cell now lies at $\sim 25 \%$. This impressive efficiency is not far behind the theoretical $\sim 33 \%$ limit calculated by Shockley and Queisser. ${ }^{2}$ This efficiency limit mainly originates from the inability of a solar cell to utilize the full solar spectrum; in particular the photons which are higher in energy than the bandgap of the semiconductor. When these photons are absorbed by the semiconductor, the resulting high-energy electronic states (so called "hot carriers") rapidly relax to the bandedge of the material through carrier-carrier scattering and carrier-phonon coupling. In principle, these hot carriers could be extracted from the semiconductor before they completely relax. ${ }^{3}$

The desire to utilize hot carriers in perovskite-based semiconductors has prompted a concerted effort to understand the parameters which control carrier relaxation. Using ultrafast laser spectroscopy, early reports by Price et al. and Yang et al. demonstrated that the cooling in methylammonium lead iodide was substantially slowed at high carrier densities. ${ }^{4,5}$ This slowed cooling can be explained through a so-called "phonon bottleneck" mechanism, where hot carriers must compete for a finite number of phonon modes in order to cool. ${ }^{6}$ In our previous work on hot carrier cooling in perovskites, we demonstrated that this phonon bottleneck behavior is sensitive to the material composition. Namely, the additional phonons associated with the organic cation in the

Corresponding author email: a.bakulin@imperial.ac.uk

Physical Chemistry of Semiconductor Materials and Interfaces XVIII, edited by Christian Nielsen, Daniel Congreve, Hugo A. Bronstein, Felix Deschler, Proc. of SPIE Vol. 11084, 110840F

(C) 2019 SPIE $\cdot$ CCC code: $0277-786 X / 19 / \$ 21 \cdot$ doi: $10.1117 / 12.2528131$

Proc. of SPIE Vol. 11084 110840F-1 
hybrid material, with comparison to the all-inorganic material, were shown to accelerate the cooling dynamics. ${ }^{7}$ The phonon bottleneck behavior is expected to be further amplified in semiconductor nanostructures, due to the quantization of electronic states. ${ }^{3}$ Early work on CdSe showed a reduced carrier cooling rate for strongly confined quantum dots with respect to the bulk material. ${ }^{8}$ On a similar note, preliminary studies by Li et al. indicated that cooling in $\mathrm{MAPbBr}_{3} \mathrm{NCs}$ was slower for smaller NCs, ${ }^{9}$ however other works have disputed the strong size-dependence of cooling in perovskite based materials. ${ }^{10,11}$

In this contribution, we use pump-push-probe spectroscopy to compare the carrier cooling dynamics in bulk and nanocrystal (NC) lead bromide perovskites with different material composition, NC diameter and surface treatment. We find that the density-dependent cooling behavior is intrinsic to the material composition and exhibits very little effect on the NC size or surface ligands, which rule out the important roles of quantum confinement and surface defects on the carrier cooling behavior. We instead find that the main parameter which controls cooling is the cation in the perovskite, as evidenced by the faster overall cooling in the hybrid $\mathrm{FAPbBr}_{3}$ $\mathrm{NCs}$ with respect to the all-inorganic $\mathrm{CsPbBr}_{3} \mathrm{NCs}$. These observations underline the unique electronic and phononic properties of perovskites and their implications towards prospective hot carrier applications.

\section{EXPERIMENTAL METHODS}

\subsection{Sample preparation and characterization}

Glass substrates were treated with $\mathrm{O}_{2}$ plasma to improve wetting. The bulk $\mathrm{CsPbBr}_{3}$ film was then spin-coated

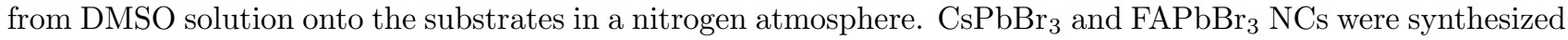
using a known procedure. ${ }^{12}$ The concentrated inks were dropcast from toluene solutions onto the glass substrates. The size of the NCs were determined by transmission electron microscopy. All film thicknesses were determined to be $\sim 300 \mathrm{~nm}$ by spectroscopic ellipsometry.

\section{$2.2 \mathrm{UV}-\mathrm{V}$ is spectroscopy}

Absorption spectra of the thin films were obtained from a UV-Vis spectrometer (Shimadzu 2600, equipped with an ISR-2600Plus integrating sphere). A $1 \mathrm{~nm}$ sampling interval was used with a $5 \mathrm{~nm}$ slit width.

\subsection{Visible pump-IR push-IR probe spectroscopy}

Two optical parametric amplifiers (TOPAS-Prime, Coherent) were seeded with $800 \mathrm{~nm}$ pulses from a Ti:sapphire regenerative amplifier (Astrella, Coherent) at a repetition rate of $4 \mathrm{kHz}$ and pulse duration of $\sim 35 \mathrm{fs}$. The signal output $(\sim 1300 \mathrm{~nm})$ of one of the optical parametric amplifiers was directed into a $\beta$-barium borate crystal (EKSMA Optics) along with the residual part of the fundamental $(800 \mathrm{~nm})$ from the regenerative amplifier. Sum frequency generation yielded the $500 \mathrm{~nm}$ pump pulse. The spectrum of the pump was measured using a spectrometer (OCEAN-FX-VIS-NIR, Ocean Optics). The idler output ( $2000 \mathrm{~nm})$ of the other optical parametric amplifier was split in a 9:1 ratio. The more intense portion was used as the push, and the weaker portion was used as the probe. The pump and probe beams were focused onto a $\sim 0.2 \mathrm{~mm}$ diameter spot on the sample, and the push was slightly defocused to facilitate beam overlap and prevent photodegradation of the sample. The transmission of the probe through the sample was recorded with an amplified PbSe photodetector (PDA20H-EC, ThorLabs). This was connected to a lock-in amplifier (SR830, Stanford Research Systems) coupled to a chopper in the pump beam which was set to a frequency of $2 \mathrm{kHz}$ to block every other pump pulse. The delay between the pump, push and probe beams were controlled with mechanical delays stages. The sample was kept in a nitrogen-purged quartz cuvette during measurements.

\section{EXPERIMENTAL RESULTS}

The linear absorption spectra for all the samples were determined by UV-Vis absorption spectroscopy, as shown below in Figure 1. 


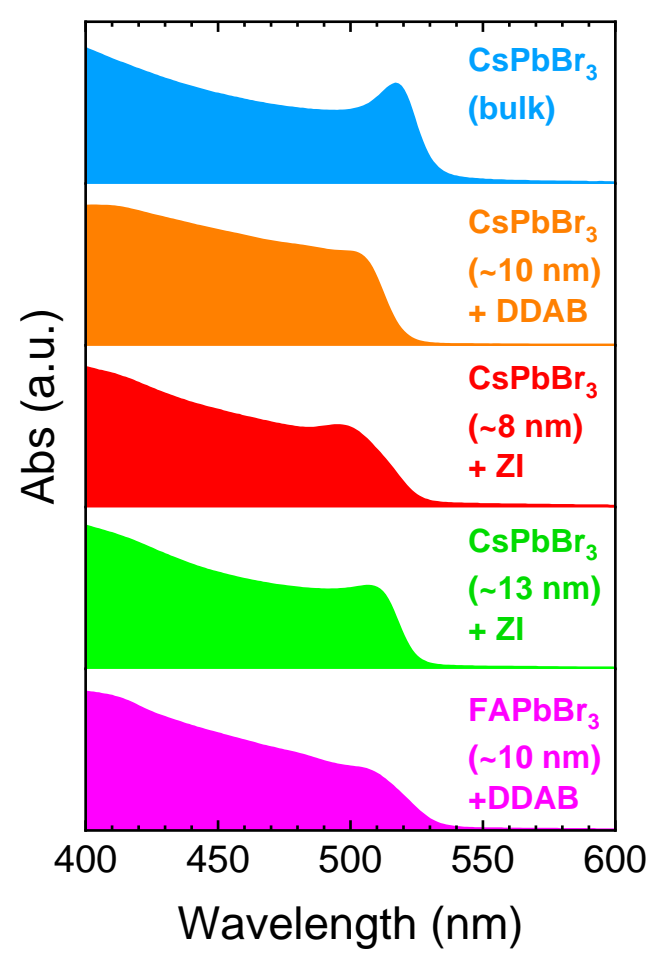

Figure 1. UV-Vis absorption spectra for all the thin film samples.

The cooling dynamics of the bulk and NC samples were determined by visible pump-IR push-IR probe spectroscopy. ${ }^{7,8,13}$ Based on the UV-Vis absorption spectra in Figure 1, a $500 \mathrm{~nm}$ pump pulse was selected to avoid excessive heating of the initial carriers. The intensity of the pump was restricted to prevent the influence of carrier-carrier interactions on the cooling process. Representative fluence-dependent pump-probe kinetics for a $\mathrm{CsPBr}_{3} \mathrm{NC}$ sample are shown in Figure 2. For the three-pulse measurements, the pump fluence was set to avoid the many-body Auger kinetics which can be seen at early timescales for higher pump intensities. ${ }^{14}$

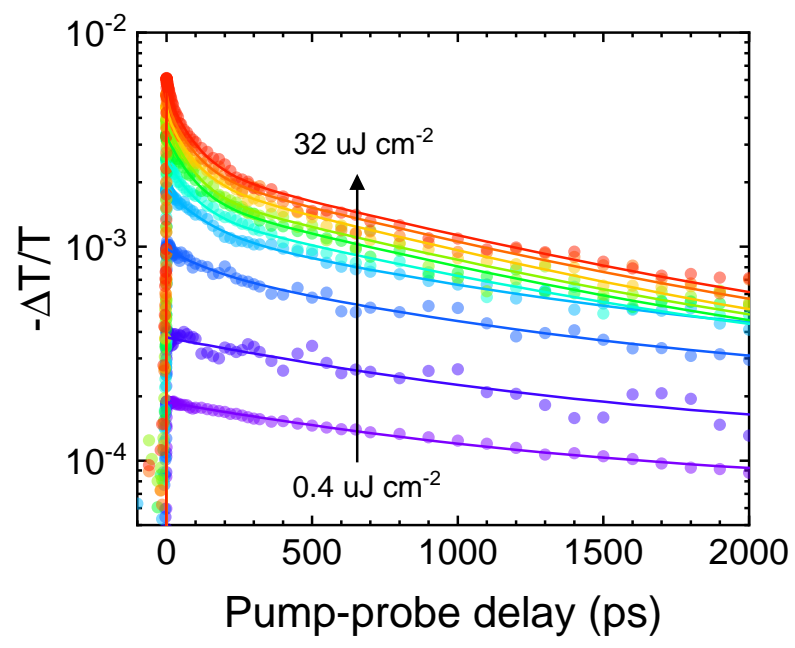

Figure 2. Fluence-dependent pump-probe kinetics for the ZI ligand-capped $\sim 8 \mathrm{~nm}$ diameter $\mathrm{CsPbBr}_{3}$ dropcast $\mathrm{NCs}$. Pump: $500 \mathrm{~nm}$; probe: $2000 \mathrm{~nm}$. Solid lines are multiexponential fits as a guide to the eye. Fluence is reported in units of $\mu \mathrm{J} \mathrm{cm}^{-2}$. 
The pump-push-probe experiment is depicted in Figure 3. Figure 3(a) shows that an intense $2000 \mathrm{~nm}$ push pulse arrives $12 \mathrm{ps}$ after the pump. The push reheats the cold carriers formed by the pump, causing a rapid bleaching of the probe signal, as shown in Figure 3(b). The probe signal recovers on the sub-ps timescale as the carriers cool back to the band edge. We fit the recovery of the signal with a monoexponential curve to extract the cooling lifetime of hot carriers $\left(\tau_{\text {cool }}\right)$.
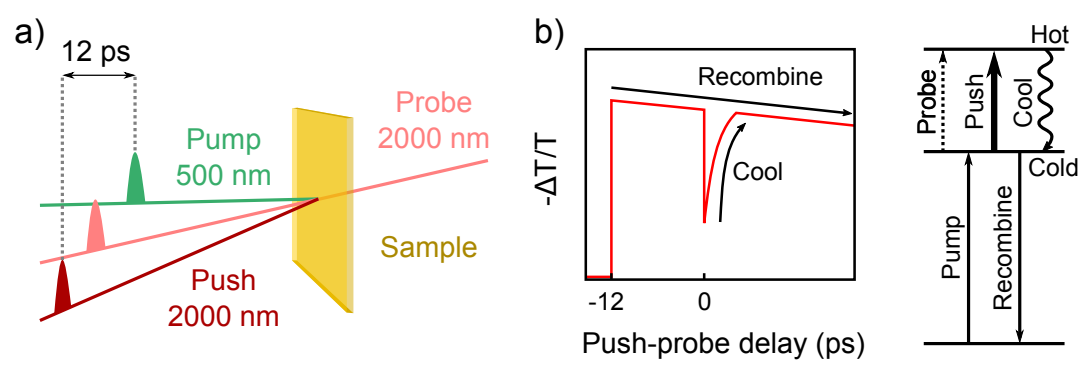

Figure 3. (a) Schematic for the three-pulse experiment. (b) Left: schematic for the differential probe transmission in response to the pump and push pulses; right: three-level model used to interpret the signal.

Figure 4 presents the cooling dynamics for all the samples under study. To facilitate the comparison between the data sets, $\tau_{\text {cool }}$ is shown as a function of the number of hot carriers formed by the push, $n_{\text {hot }}$, relative to the number of cold carriers created by the pump, $n_{\text {cold }} . n_{\text {cold }}$ is calculated from the absorption cross section of the sample, while $n_{h o t}$ is estimated from the ratio between the amplitude of the bleach feature at $\mathrm{t}=0$ and the amplitude of the pump-probe signal before $\mathrm{t}=0$.
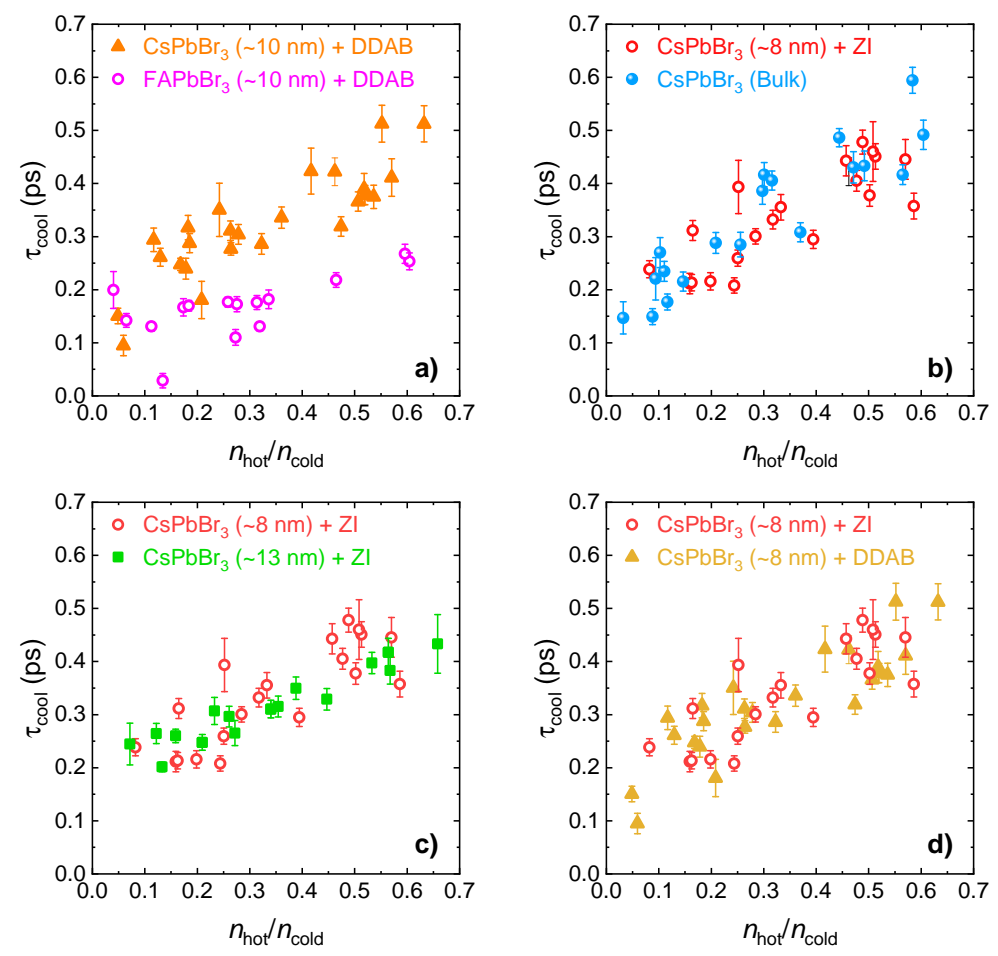

Figure 4. Density-dependent carrier cooling dynamics for the systems under investigation. (a) compares the dynamics for $\mathrm{CsPbBr}_{3}$ and $\mathrm{FAPbBr}_{3} \mathrm{NCs}$; (b) compares the dynamics for bulk and $\mathrm{NC}$-based $\mathrm{CsPbBr}_{3}$; (c) compares the dynamics for two sizes of $\mathrm{CsPBBr}_{3} \mathrm{NCs}$ capped with ZI ligand; (d) compares the dynamics for two $\mathrm{CsPbBr}_{3} \mathrm{NCs}$ capped with different surface ligands. 


\section{DISCUSSION}

\subsection{Effect of hot carrier density}

For all the cases in Figure 4, the lifetime of the hot carriers is shown to increase with an increasing density of hot carriers. This underlying behavior is attributed to a so-called "phonon bottleneck" mechanism, where carriers must compete for a finite number of vibrational modes (phonons) in order to cool. ${ }^{6}$ This effect has been reported in numerous lead-halide perovskite materials. ${ }^{4,5,7,15}$

\subsection{Effect of the cation}

As shown in Figure 4(a), the choice of cation in the lead-halide perovskite has a strong effect on the cooling of the hot carrier ensemble. In our previous work on bulk lead-halide perovskites, we complemented ultrafast measurements with quantum chemical calculations to demonstrate that the density of phonon modes associated with the cation is the key material factor which determines the cooling of the hot carriers. With respect to the complex formamidinium (FA) cation, the symmetric and isotropic cesium (Cs) cation possesses fewer phonon modes into which the excess energy of the hot carriers can be deposited, resulting in overall slower cooling of the hot carriers. ${ }^{7}$ The observation of slower carrier cooling in the all-inorganic perovskites has also been reported elsewhere. ${ }^{11,16-18}$

\subsection{Effect of weak quantum confinement}

Figure 4(b) shows that switching from bulk $\mathrm{CsPBBr}_{3}$ to the analogous NC sample has very little effect on the overall cooling behavior of the hot carriers. Likewise, changing the size of the nanocrystal in Figure 4(c) appears to have no observable effect on the cooling rate either. The UV-Vis absorption spectra of these samples in Figure 1 highlight the fact that the perovskite NCs are only weakly quantum confined; the absorption onset, i.e. bandgap of the materials, is not strongly blueshifted with respect to the bulk materials. Such weak quantum confinement of the electronic states is not expected to strongly modify the specific nature of the electron-phonon coupling in these materials. The small dependence of the carrier cooling on NC size in these weakly confined materials has been reported in other works. ${ }^{10,11}$

\subsection{Effect of surface states}

The similarity in the cooling dynamics of the bulk and $\mathrm{NC}$-based $\mathrm{CsPbBr}_{3}$ samples of different sizes suggests that the different surface areas and extent of material interfaces do not play a significant role in the cooling dynamics of the hot carriers for the lead-bromide perovskites. Along the same vein, Figure 4(d) shows that the cooling behavior in NC samples capped with didodecyl dimethylammonium bromide (DDAB) or the superior zwitterionic (ZI) ligands ${ }^{12}$ is identical, which appears to preclude the effect of any surface-bound defects on the relaxation of the hot carriers. These observations are in contrast to reports from other colloidal NCs measured with a similar technique, where the nature of the surface states plays an important role in the cooling of hot carriers. ${ }^{8}$ It is worth noting that the electronic properties of lead-halide perovskites are known to have a greater tolerance towards defects than traditional $\mathrm{NC}$ materials. ${ }^{19}$

\section{CONCLUSION}

Using pump-push-probe spectroscopy, we compare the hot carrier cooling dynamics in bulk and nanocrystal (NC) samples of lead-bromide perovskite with different material composition, NC diameter and surface treatment. For all samples, we find that the carrier cooling becomes slower with an increasing number of hot carriers, which we attribute to a phonon bottleneck mechanism. This density-dependent cooling behavior appears to be intrinsic to the material composition rather than size or surface effects, in contrast to traditional colloidal NCs where quantum confinement and defect states have been shown to play an important role in the hot carrier relaxation process. In agreement with our previous studies on bulk lead-halide perovskites, we instead find that the main material determinant in the cooling dynamics appears to be the choice of the cation, as evidenced by the faster overall cooling in the hybrid $\mathrm{FAPbBr}_{3} \mathrm{NCs}$ with respect to the all-inorganic $\mathrm{CsPbBr}_{3} \mathrm{NCs}$. These observations underline the unique electronic and phononic properties of lead-halide perovskites and their implications towards prospective hot carrier applications. 


\section{ACKNOWLEDGMENTS}

A.A.B. is a Royal Society University Research Fellow.

\section{REFERENCES}

[1] NREL, "Best Research-Cell Efficiencies Chart." NREL, 08 February 2019 https://www.nrel.gov/pv/ assets/pdfs/best-research-cell-efficiencies.20190802.pdf. (Accessed: 27 August 2019).

[2] Shockley, W. and Queisser, H. J., "Detailed Balance Limit of Efficiency of pn Junction Solar Cells," Journal of Applied Physics 32(3), 510-519 (1961).

[3] Nozik, A. J., "Spectroscopy and Hot Electron Relaxation Dynamics in Semiconductor Quantum Wells and Quantum Dots," Annual Review of Physical Chemistry 52(1), 193-231 (2001).

[4] Price, M. B., Butkus, J., Jellicoe, T. C., Sadhanala, A., Briane, A., Halpert, J. E., Broch, K., Hodgkiss, J. M., Friend, R. H., and Deschler, F., "Hot-carrier cooling and photoinduced refractive index changes in organicinorganic lead halide perovskites," Nature Communications 6(1), 8420 (2015).

[5] Yang, Y., Ostrowski, D. P., France, R. M., Zhu, K., van de Lagemaat, J., Luther, J. M., and Beard, M. C., "Observation of a hot-phonon bottleneck in lead-iodide perovskites," Nature Photonics 10(1), 53-59 (2016).

[6] Frost, J. M., Whalley, L. D., and Walsh, A., "Slow Cooling of Hot Polarons in Halide Perovskite Solar Cells," ACS Energy Letters 2(12), 2647-2652 (2017).

[7] Hopper, T. R., Gorodetsky, A., Frost, J. M., Müller, C., Lovrincic, R., and Bakulin, A. A., "Ultrafast Intraband Spectroscopy of Hot-Carrier Cooling in Lead-Halide Perovskites," ACS Energy Letters 3(9), 2199-2205 (2018).

[8] Guyot-Sionnest, P., Shim, M., Matranga, C., and Hines, M., "Intraband relaxation in CdSe quantum dots," Physical Review B 60(4), R2181-R2184 (1999).

[9] Li, M., Bhaumik, S., Goh, T. W., Kumar, M. S., Yantara, N., Grätzel, M., Mhaisalkar, S., Mathews, N., and Sum, T. C., "Slow cooling and highly efficient extraction of hot carriers in colloidal perovskite nanocrystals," Nature Communications 8(May), 14350 (2017).

[10] Butkus, J., Vashishtha, P., Chen, K., Gallaher, J. K., Prasad, S. K. K., Metin, D. Z., Laufersky, G., Gaston, N., Halpert, J. E., and Hodgkiss, J. M., "The Evolution of Quantum Confinement in CsPbBr 3 Perovskite Nanocrystals," Chemistry of Materials 29(8), 3644-3652 (2017).

[11] Diroll, B. T. and Schaller, R. D., "Intraband Cooling in AllInorganic and Hybrid OrganicInorganic Perovskite Nanocrystals," Advanced Functional Materials 1901725, 1901725 (2019).

[12] Krieg, F., Ochsenbein, S. T., Yakunin, S., ten Brinck, S., Aellen, P., Süess, A., Clerc, B., Guggisberg, D., Nazarenko, O., Shynkarenko, Y., Kumar, S., Shih, C.-J., Infante, I., and Kovalenko, M. V., "Colloidal CsPbX $3(\mathrm{X}=\mathrm{Cl}, \mathrm{Br}, \mathrm{I})$ Nanocrystals 2.0: Zwitterionic Capping Ligands for Improved Durability and Stability," ACS Energy Letters 3(3), 641-646 (2018).

[13] Rabouw, F. T., Vaxenburg, R., Bakulin, A. A., van Dijk-Moes, R. J. A., Bakker, H. J., Rodina, A., Lifshitz, E., L. Efros, A., Koenderink, A. F., and Vanmaekelbergh, D., "Dynamics of Intraband and Interband Auger Processes in Colloidal CoreShell Quantum Dots," ACS Nano 9(10), 10366-10376 (2015).

[14] Makarov, N. S., Guo, S., Isaienko, O., Liu, W., Robel, I., and Klimov, V. I., "Spectral and Dynamical Properties of Single Excitons, Biexcitons, and Trions in CesiumLead-Halide Perovskite Quantum Dots," Nano Letters 16(4), 2349-2362 (2016).

[15] Yang, J., Wen, X., Xia, H., Sheng, R., Ma, Q., Kim, J., Tapping, P., Harada, T., Kee, T. W., Huang, F., Cheng, Y.-B., Green, M., Ho-Baillie, A., Huang, S., Shrestha, S., Patterson, R., and Conibeer, G., "Acousticoptical phonon up-conversion and hot-phonon bottleneck in lead-halide perovskites," Nature Communications 8, 14120 (2017).

[16] Madjet, M. E., Berdiyorov, G. R., El-Mellouhi, F., Alharbi, F. H., Akimov, A. V., and Kais, S., "Cation Effect on Hot Carrier Cooling in Halide Perovskite Materials," The Journal of Physical Chemistry Letters 8(18), 4439-4445 (2017).

[17] Bretschneider, S. A., Ivanov, I., Wang, H. I., Miyata, K., Zhu, X., and Bonn, M., "Quantifying Polaron Formation and Charge Carrier Cooling in Lead-Iodide Perovskites," Advanced Materials 0(0), 1707312 (2018). 
[18] Chen, J., Messing, M. E., Zheng, K., and Pullerits, T., "Cation Dependent Hot Carrier Cooling in Halide Perovskite Nanocrystals," Journal of the American Chemical Society, jacs.8b11867 (2019).

[19] Akkerman, Q. A., Rainò, G., Kovalenko, M. V., and Manna, L., "Genesis, challenges and opportunities for colloidal lead halide perovskite nanocrystals," Nature Materials 17(5), 394-405 (2018). 